\title{
Eukaryo: An AR and VR Application for Cell Biology
}

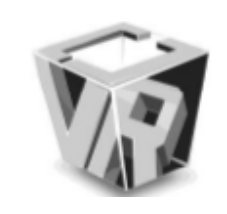

\author{
Douglas Yuen ${ }^{1}$, Markus Santoso ${ }^{1}$, Stephen Cartwright ${ }^{2}$ and Christian Jacob ${ }^{3}$
}

${ }^{1}$ Dept. of Computer Science, University of Calgary

${ }^{2}$ Simulation and Visualization, FCMG Frank and Sarah Meyer Collaboration Centre, University of Calgary

${ }^{3}$ Dept. of Computer Science, Dept. of Biochemistry \& Molecular Biology, University of Calgary

\begin{abstract}
Eukaryo is a simulated bio-molecular world that allows users to explore the complex environment within a biological cell. Eukaryo was developed using Unity, leveraging the capabilities and high performance of a commercial game engine. Through the use of MiddleVR, our tool can support a wide variety of interaction platforms including 3D virtual reality (VR) environments, such as head-mounted displays, augmented reality (AR) headsets, and large scale immersive visualization facilities. Our interactive, 3-dimensional model demonstrates key functional elements of a generic eukaryotic cell. Users are able to use multiple modes to explore the cell, its structural elements, its organelles, and some key metabolic processes. In contrast to textbook diagrams and even videos, Eukaryo immerses users directly in the biological environment, giving a more effective demonstration of how cellular processes work, how compartmentalization affects cellular functions, and how the machineries of life operate. .
\end{abstract}

Index Terms - Virtual reality; augmented reality; biological simulation; 3D game engine; cell metabolism; machinery of life; eukaryotic cell.

\section{Introduction}

Notions of scaling humans down to a molecular size so that we may explore biological systems on a cellular level have long been constrained to the realm of science fiction. In the 1966 film "Fantastic Voyage" $(9$, ?) a group of doctors and scientists navigates through the human body in a miniaturized submarine. If actually possible, such journeys would provide an unparalleled experience for medical researchers and students alike. David Goodsell's illustrations in his book "The Machinery of Life" captures tissues and cells in high detail at the molecular level by making use of the latest imaging techniques (13). Intended to provide a glimpse into the complex forms and organization of these tissues and cells, Goodsell's illustrations are meticulously constructed from a combination of high-resolution electron micrographs as well as NMR and X-ray crystallography. The densely packed renderings demonstrate that the spaces within a cell are far from empty, which is contrary to the impressions that one might get from a typical textbook illustration of a cell

\footnotetext{
E-mail: dwkyuen@ucalgary.ca
}

(14). Goodsell suggests that all of the structures visible in the illustrations are probably regulated and supported by a plethora of other structures.

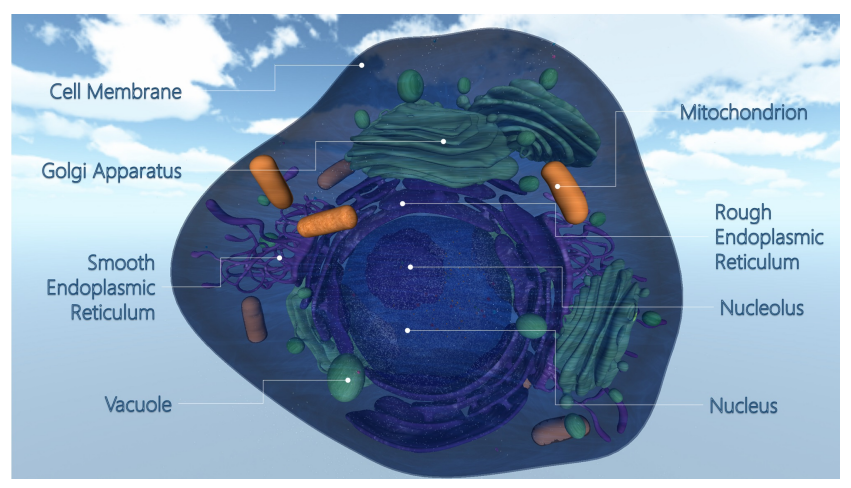

Figure 1: View of the Eukaryo eukaryotic cell model with labelled organelles. Each of these membrane-bound compartments is given a distinct colour to indicate its function.

While Goodsell's renderings capture the density of materials within cells and tissues, they are still static and thus unable to depict how the interactions among different structures contribute to biological function. Consequently, textbooks and illustrations tend to be supplemented with videos that illustrate the progression of different reactions and pathways within such a complex system. Using 3D computer animation, the BioVisions video "Inner Life of a Cell" strives to depict a variety of cellular processes that range from gene expression to molecular transport (3). However, videos only illustrate events from a fixed perspective and do not allow any further exploration or interaction with a model. Further to this, moving between different media such as illustrations and videos disrupts the continuity of the learning experience.

In order to enable interactive investigations of the machinery of life, we have implemented Eukaryo, a virtual eukaryotic cell (Fig. 1). We have implemented our cell model in the Unity game development environment (42). Using Unity gives us access to the features and high performance rendering and simulation support available in commercial game engines. We also use MiddleVR for Unity (33) to abstract some of the display and interaction methods. This allows our tool to take advantage of a wide variety of visualization 


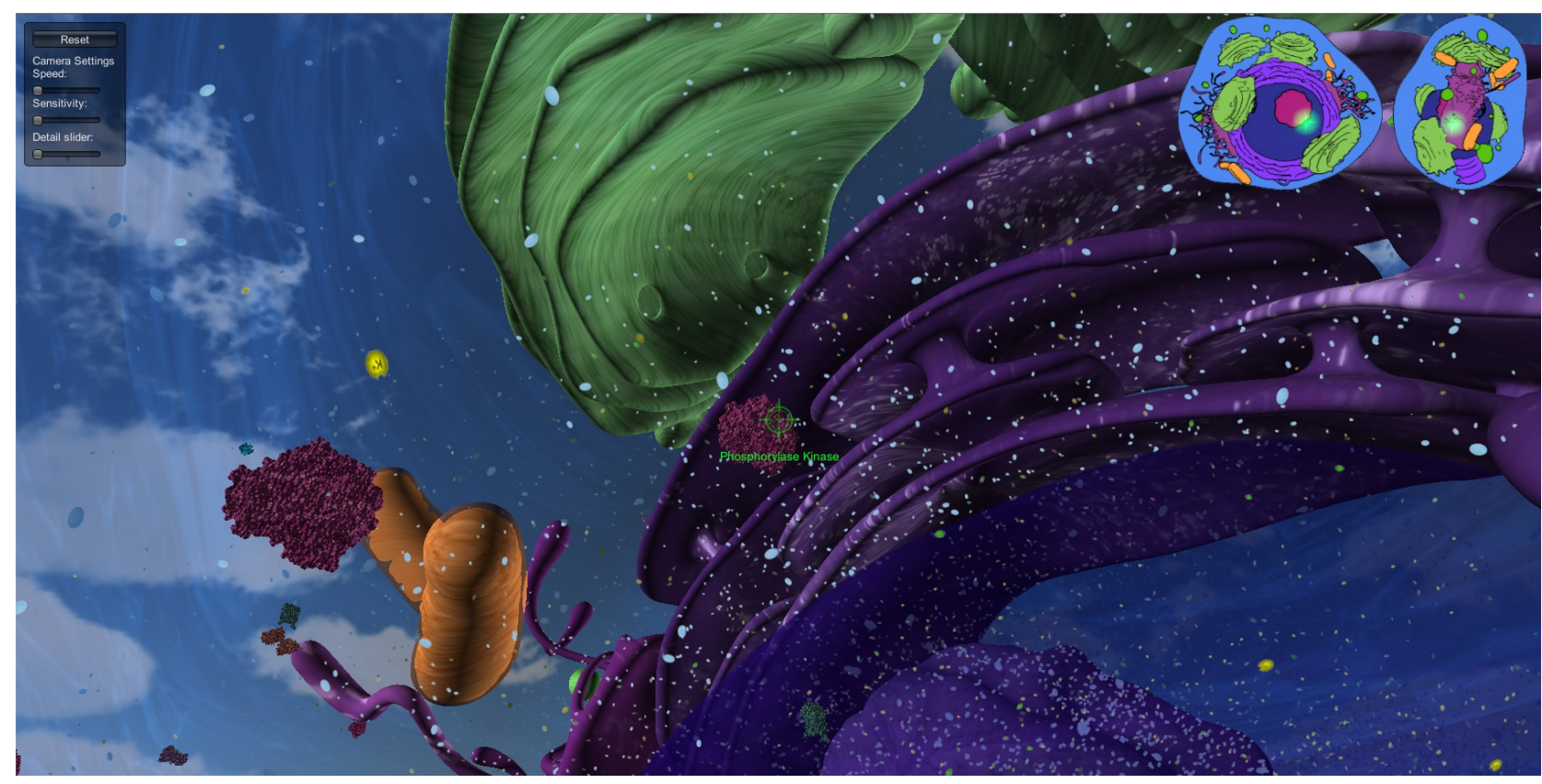

Figure 2: A view of our Eukaryo cell model with all of the HUD components visible: One sets parameters through the control panel in the top left corner. The minimap in the top right corner gives an overview of the cell space as a top-down and side view; the current camera location is indicated by a highlight.

platforms other than traditional monitors, including virtual reality platforms like the Oculus Rift head mounted display (35), immersive CAVEs (6), or augmented reality displays such as the Hololens (32).

By combining the interactivity of a video game with the immersive experience provided by virtual reality, Eukaryo allows users to explore the complex spaces within a cell in a manner reminiscent of "The Fantastic Voyage" cinematographic adventure. Not only is this a novel and engaging learning approach, this experience also provides a more effective method for visualization and understanding of the actual biological model (25). As a user, being directly transported to the interior of a cell, one can better visualize the different structures and gain a sense of the amount of activity within a "cell universe", something illustrations and videos cannot adequately capture. Although we are not yet capable of scaling ourselves down to travel into a micro-biological environment, for now we can make use of gaming tools and technologies such as VR to reproduce an experience similar to and perhaps even more effective than compelling stories and movies, such as the "Fantastic Voyage."

Works such as (24) and (?) have shown that immersion provides benefit when performing visual analysis tasks with volumetric and spatial data. Ragan et al. have elucidated the advantages of field of regard, head tracking and stereoscopic 3D on a small scale judgment task (38). The advantages of large scale immersive environments, in particular, have been highlighted by Kuhlen and Hentschel (22). These advantages help with the understanding and insight of the subject matter, in our case the complex universes within a biological cell.

\section{Related Work}

Developments in computer simulations have historically been closely tied to advances in computer technology: as the hardware improves, so does the complexity of computer models. Increased versatility and capability of software tools has made it appealing to apply computer modeling to the simulation of biological systems. While biological systems are ideally studied in vivo, most processes often cannot occur outside of a living system, thus making them difficult to study. Computer models overcome this constraint, allowing for individual processes to be observed in greater detail. As such, these models become a powerful means of simulating and illustrating cells and their processes in great detail.

Cells. Constituting the foundational building block of any biological system, simulations of cells are of particular interest. Projects such as E-CELL (41) and Virtual Cell (28) provide the frameworks for modelling interconnected processes inside a biological cell. E-CELL was implemented in 1998 and could simulate signaling, cellular reactions and gene regulation, while Virtual Cell was intended to act as a more general platform for simulations of micro-biological systems.

However, both E-CELL and Virtual Cell produce numerical outputs whose meanings are not immediately apparent to the general user. Moreover, these models are limited in their interactivity: while they are running, users cannot visually observe a process or pause the simulation to inspect its current state.

Molecular Dynamics. Works such as UnityMol (29) and MolecularRift (34) have been developed using Unity to visualize molecules. MolecularRift works on the Oculus Rift, 
whereas UnityMol has some virtual reality support. These works further demonstrate the applicability of Unity as a development tool and the benefit of virtual reality to molecular visualization, however they are focused on molecular dynamics and drug design applications rather than full cell simulations.

Agent-based Modeling. Agent-based methods have been used as simulation techniques complementary to purely mathematical models (16). More recently, a gene regulatory model of the $\lambda$-switch has been recreated in a $3 \mathrm{D}$, agent-driven simulation (19). A classic gene regulation model studied in $E$. coli bacteria, the lactose operon, has been implemented to illustrate the protein interactions that determine gene expression $(17 ; 4,18)$. In comparison to other simulators, LINDSAY Composer offers a 3D, interactive environment where models can be directly constructed in virtual, 3-dimensional spaces (20). The feasibility of such agent-based models in LINDSAY Composer has been demonstrated in an immune system simulation, purely based on interacting components, that represent various entities involved in immunological processes (40) or gene expression in E. coli (8). LINDSAY Composer has been our first attempt at combining agent-based modeling with a simulation and development environment inspired by game engines. With the advent of and convenient access to commercial game engines, we discontinued our own game engine development and focussed our efforts instead on rebuilding our biological models in Unity 3D (42) and Unreal Engine (11). Using professional game engines enables us to focus on the actual scientific contents and visual presentation of our simulations, rather than having to build, update, and maintain our own engine for a range of different computing platforms.

\section{Eukaryotic cells}

Eukaryotic cells differ from prokaryotic cells in that they are much larger in size. Their interiors are subdivided into distinct, membrane-bound compartments known as organelles, that carry out specific functions within the cell (Fig. 1) (1; ?). Of the organelles, the nucleus is the most distinct structure within a cell. The nucleus houses the DNA as well as proteins necessary to maintain the genes' integrity and to regulate transcription, which is making a copy of DNA sections into messenger RNA (mRNA). Contrary to the cell, the interior of the nucleus is not subdivided by membranes. Besides the nucleus, all eukaryotic cells have organelles for transport (Golgi apparatus and endoplasmic reticulum), storage (vesicles), metabolism (mitochondria), and maintenance of structural integrity (cytoskeleton, centrioles). Organelles within the eukaryotic cell are held together by a gel-like matrix known as the cytosol; materials and the organelles themselves can move through this matrix.

By separating different regions and their materials from one another, eukaryotic cells can closely control their processes: an extensive network of organelles within a eukaryotic cell are dedicated towards transporting and storing materials. Similarly, processes such as metabolism and transcription become more elaborate in eukaryotes. This makes it necessary for the cell to regulate the movement of different materials between regions of the cell. This is in contrast to prokaryotic (bacterial) cells, where cellular processes occur entirely in the cell's cytosol. Because of their greater surface area-to-volume ratio, reactions in prokaryotic cells can occur more quickly and efficiently without additional regulatory mechanisms, but this also precludes more complex functions.

Compartmentalization in eukaryotic cells have allowed for more complex organisms to arise. Yet the greater intricacies of a eukaryotic cell makes it challenging to study specific pathways and molecular interactions within a cell. This is the reason why prokaryotes, such as E. coli, are much better characterised than their more complex eukaryotic counterparts. Hence, it becomes even more important to provide accessible, engaging, and highly visual computer models that help to shed more light on the inner workings of such cellular machineries. Furthermore, as our bodies are constructed from eukaryotic cells, intricate cell models are of great importance to medical research and medical education, in particular.

In this paper, after explaining the key cellular components implemented in our model, we explore two cellular processes in greater detail: nuclear export of messenger RNA into the cytosol from the nucleus, and the dynamics of microtubule assembly as part of the cell cytoskeleton. Through these examples, we illustrate how virtual reality technology is used to simulated and visualize dynamic processes in cell biology.

\section{Implementing Eukaryo}

In contrast to previous cell simulations, including LINDSAY Composer, we have built Eukaryo within the Unity (42) game engine. The Eukaryo model consists of a collection of game objects, which act as containers for components. With very specific functions, such components can be used in varying combinations to build distinct game objects. In our case, these game objects constitute cell components and their visual elements, as well as control components that handle VR rendering, navigation, and head-up displays (Fig. 2). Together, these game objects create an immersive $3 \mathrm{D}$ space that replicates the environment within a biological cell. Despite the amount of activity that is rendered in $E u$ karyo, the model runs smoothly both in the CAVE and with the Oculus Rift headset.

\section{The Cell Components}

The initial graphical assets were provided by Zygote Media Group (46) and given textures using Autodesk Maya (30) such that the organelles - subunits within the cell that carry out specific functions - are easily differentiable from one another (Fig. 1). The nucleus houses DNA and is the site of mRNA transcription (12). The rough endoplasmic reticulum is a site for protein synthesis, while the smooth endoplasmic reticulum handles lipid synthesis and calcium regulation. These structures are colored with shades of purple. The Golgi apparati are depicted in green: they package proteins into membrane-bound structures known as vesicles for transport out of the cell. Vacuoles are large membranebound organelles that consist largely of water with suspended proteins and macromolecules. Since they also have 
a storage-related function, they are given a green texture. As the site of energy production in the cell, the mitochondria have an orange coloration, allowing them to stand out from other organelles. Together, the coloration makes organelles easily distinguishable and identifiable of their roles in the cell.

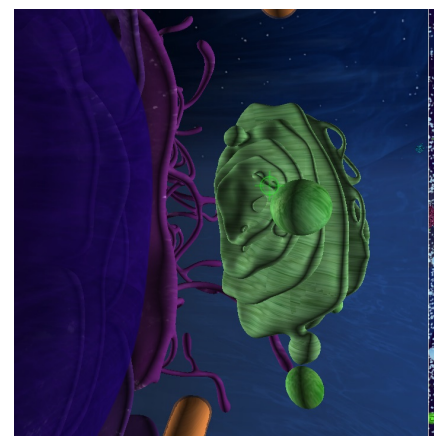

(a) Low detail

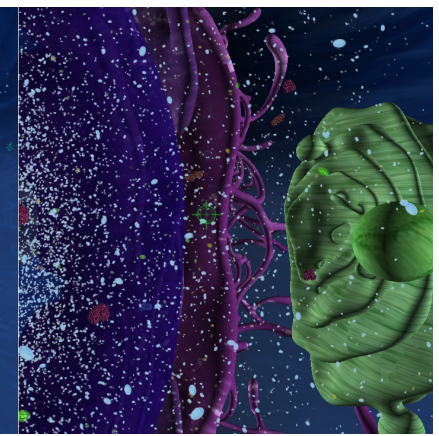

(b) High detail
Figure 3: Side-by-side comparison of the cell under different levels of detail. (a) With all game objects and particle systems disabled, the viewer can focus on the cell's structures. (b) All of the elements are visible to capture the crowded cell environment.

\section{Rendering Crowded Spaces}

To further enhance the visual impact, additional elements are incorporated to ensure that the busy environment in the cellular space is captured. We apply Unity's particle systems, which are typically used to depict transient sprites such as fire, smoke and spark effects. The particle systems in Unity can also be used to project billboards (39). These twodimensional billboard sprites always face the camera and can be customized to represent small entities present in the cytosol (Fig. 33). Multiple particle systems were added to the cell to visualize entities such as water, protons, sodium, potassium and chlorine (Fig. 3p). The crowded space inside the cytosol means that molecules move around randomly, so each particle system is configured to emit particles with variable speeds, lifetimes and angular motion. This adds to the sense that their motion is random - as is the case in real cells, where most of the movement of molecules is driven by Brownian motion.

Besides ions and molecules, proteins populate the cytosol. Because proteins participate in reactions, they are represented by individual game objects that are capable of interacting with one another: each protein game object consists of a mesh and an optional script component that defines its rules of interaction. Mesh data for each protein is obtained from the Protein Databank (PDB) (43). All proteins in our cell model have a corresponding PDB file, a specially-formatted text file that stores the coordinates and rotations of each amino acid to reflect their organizational structure. This information can be used in programs such as UCSF Chimera to visualize protein structures in high detail (36). In comparison to other PDB viewers, Chimera is able to generate a protein's surface using this information and map the surface geometries for smaller proteins. Once the 3D surfaces are rendered, they can be exported to Unity. Creating protein models in this manner offers the advantage that the mesh is able to more accurately represent a protein's structure, which can be quickly updated if new structural information becomes available.

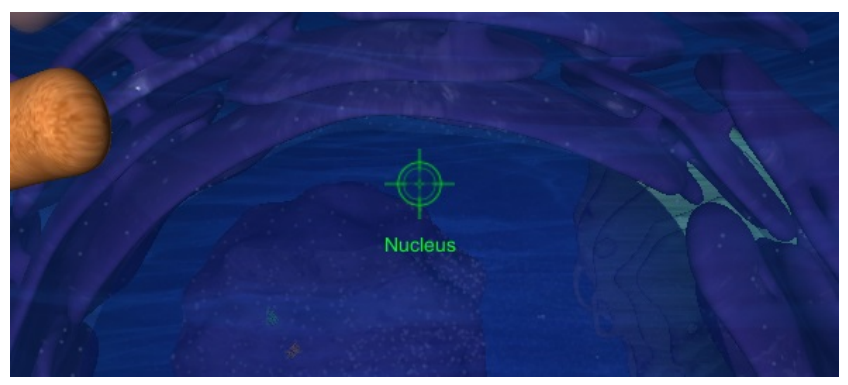

(a) Crosshair and label

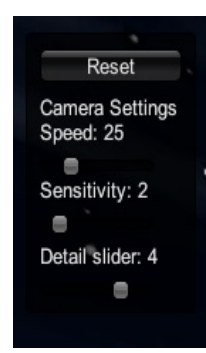

(b) Controls

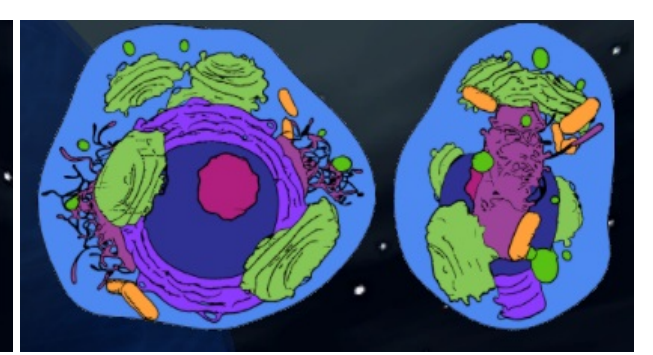

(c) Minimaps
Figure 4: Different HUD elements within Eukaryo. (a) Crosshair helps users track their gaze. (b) Movement speed, display sensitivity as well as the level of detail within the model are set through control panels. (c) A minimap indicating one's relative location inside the cell helps with orientation and navigation (see also Fig. 2.

\section{Virtual Reality and Modes of Navigation}

Eukaryo uses MiddleVR (23) so that the details of different visualization environments, such as display setup and input, do not have to be manually programmed for each display environment. With the MiddleVR interface layer, a project can be deployed to a variety of VR platforms including the CAVE and Oculus Rift, as well as technologies such as Holobench or $3 \mathrm{D}$ monitors. Once the plugin is added to a Unity project, the resulting executable can be run in many different environments. Changes then only need to be made to configuration files to define details such as display hardware and input specifications. MiddleVR's relatively simple setup and its integration with Unity makes it a powerful tool to add support for different visualization technologies such as VR to the cell model. This allows a user to take advantage of the best visualization hardware available to ensure an optimal experience of the virtual cell. A user also has flexibility to use different environments such as a CAVE (Fig. 5) for demonstrations or collaborative work as well as 
technologies such as a head mounted display for individual exploration.

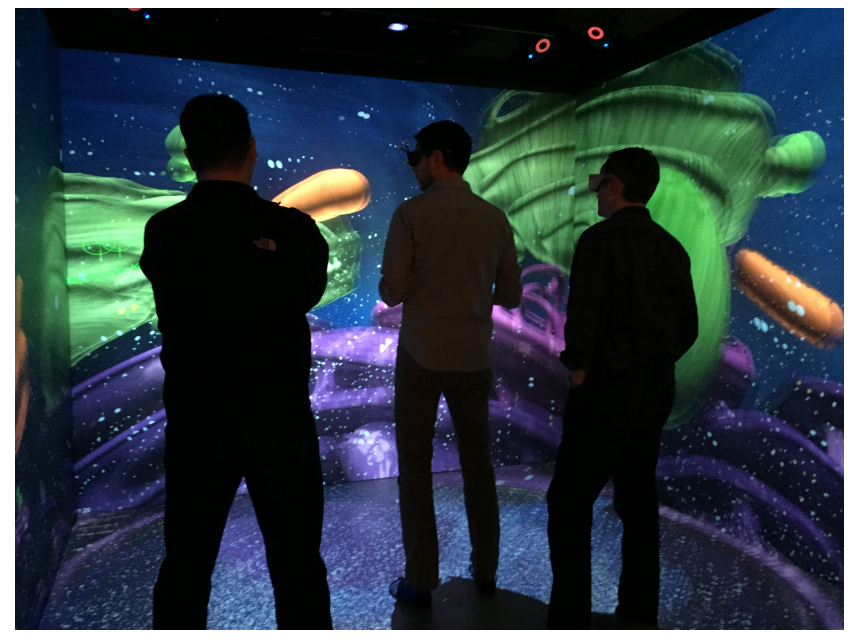

Figure 5: Eukaryo running in the CAVE. The model can be navigated through a handheld gamepad. Stereoscopic glasses create a 3D image. Motion sensor balls mounted onto the camera and glasses track the user's head and hand movements.

Having an intuitive, simple means of navigation is critical in Eukaryo. Contrary to other virtual environments that depict buildings and landscapes, there are no familiar reference points within a cell or clearly defined notions of 'up/down' or 'north/south'. It is therefore more challenging for users to orient themselves based entirely on the visual cues inside the cell. As a navigational aid, a minimap, displayed in the upper right-hand corner of the screen, provides information about one's location (Fig. 4c). Additionally, a controller for adjusting the visible level of activity inside the cell was implemented in the form of a simple slider (Fig. 4p). This allows users to hide and show different elements and processes inside the cell. At the lowest activity level setting, only the cell and its organelles are visible (Fig. 3a). At the maximum level of detail all of the molecules and proteins are depicted. Having all of these elements visible results in the most detailed view of the cellular interior (Fig. 3p). This can be visually overwhelming, especially as many of these elements are in constant motion and interact with each other. Through the detail sliders one is able to dial in just enough details for the currently observed molecular process. Whenever game objects are hidden from view, the interaction processes still continue to run in the background, so the simulation itself is never interrupted; only more or less elements are visualized.

Eukaryo can be operated by a mouse and keyboard, but through MiddleVR, Eukaryo also accepts input from a wireless gamepad controller. In the CAVE, this frees users from being constrained by cables and the need to navigate using separate input devices (Fig. 5). The wireless controller is also suitable for the Oculus Rift. Both the head-mounted display and the CAVE, use head-tracking to change camera orientation within the 3D scene. Through the controller, one can adjust the level of detail in the cell, as well as the move- ment speed of the camera. When the cell is running in a VR environment, a crosshair is linked to the head tracking input to follow the user's gaze in order to indicate what the user is looking at (Fig.4 4). This allows users to select an object and bring up more information about it. Currently, for each object a label is displayed, from which more information about the object in focus is accessible.

\section{Exploring virtual cell spaces}

Eukaryo's capacity for rendering cellular spaces in high detail provides the opportunity for users to explore biological processes in a much more open and immersive manner than previously possible. To illustrate this, we explore two cellular processes in more detail: (1) nuclear export of mRNA from the nucleus into the cytosol, and (2) the assembly and disassembly of microtubules, which are the largest filaments of the cytoskeleton. Making use of the navigational infrastructure in Eukaryo, users are able to observe and interact with these processes in a virtual environment.

\section{Nuclear Transport of mRNA}

Proteins are a cell's building blocks, which give it structure and act as functional units; they are key players for cell growth, metabolism, and communication. Proteins are encoded through DNA molecules, which reside inside the nucleus. Sections of DNA are transcribed into messenger RNA (mRNA) as an intermediary information carrier (1; ?). mRNA specifies sequences of nucleotides, which - after leaving the nucleus - are translated into chains of amino acids that fold into the 3-dimensional structures of proteins. This so-called gene expression is tightly regulated in eukaryotic cells. The two processes of transcription (from DNA to mRNA,Fig. 7) and translation (from mRNA to amino acids, Fig. 10, occur in physically separate spaces: the nucleus and the cytoplasm, respectively (Fig. 6).

Multiple processes contribute to this regulation, including the tight control of mRNA export from the nucleus into the cytoplasm (Fig. 8). This export is the process where mRNA strands are produced during transcription, which is the process where the nucleotides on DNA are read by RNA polymerase to construct a strand of complementary mRNA (Fig. 7). This newly synthesized mRNA is tagged by nuclear protein signals, allowing the nascent strands to be transported across the nuclear pore and into the cytoplasm (5). Once mRNA is synthesized, several nuclear proteins (THO, UAP56 and ALY) bind to it and recruit an export factor called Nxf1-Nxt1 (Fig. 8). The export factor allows the nuclear pore complex to recognize that the mRNA is ready to be moved out into the cytoplasm. The mRNA strand is transported across the nuclear membrane, where the export factor detaches. Subsequently, two proteins, Gle1 and Dbp5, bind to the mRNA and are the signal to begin translation: ribosome proteins read the mRNA and construct the corresponding amino acid chains that fold into a protein (Fig. 10) (5).

This process is reproduced within Eukaryo. We illustrate the sequence in which the nuclear proteins bind to mRNA. The different export factors, signalling proteins and 


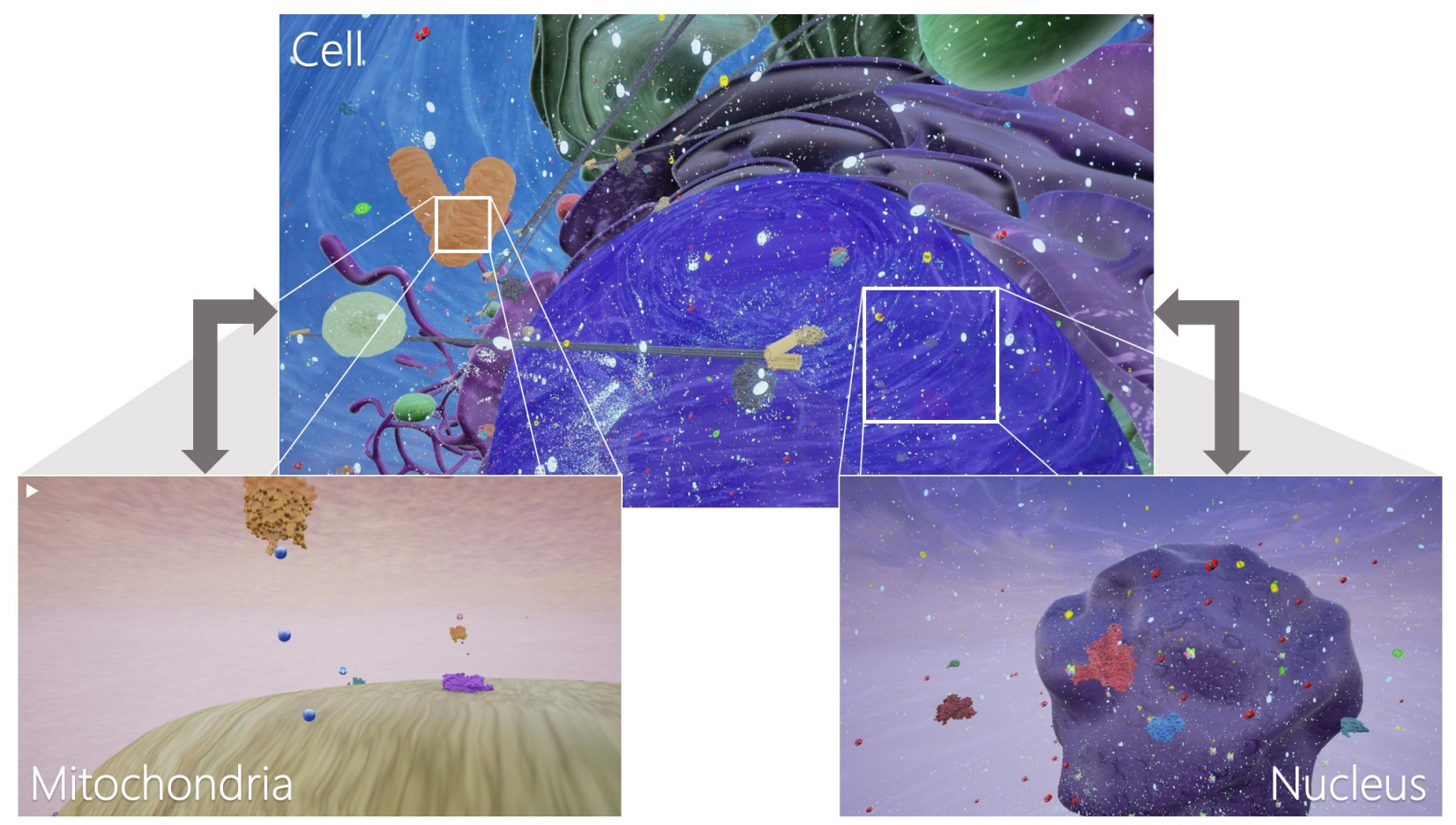

Figure 6: Setup of different scenes in Eukaryo. Users can transition from the cell level to the nucleus or mitochondria levels to view their interiors and processes in greater detail.

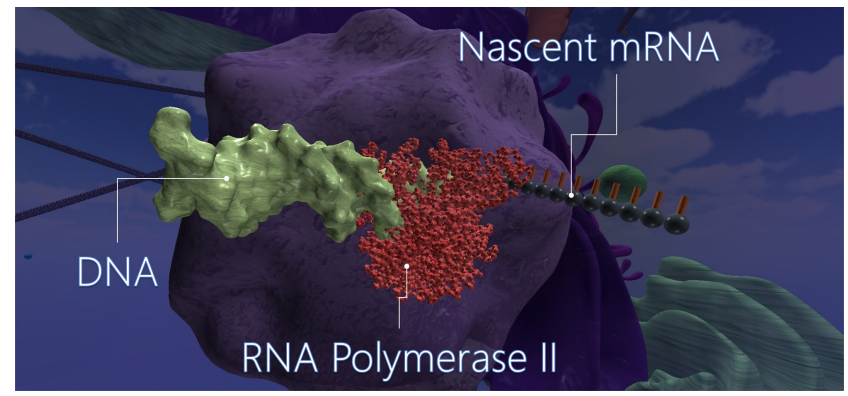

Figure 7: Transcription of RNA from DNA. This process is carried out by RNA Polymerase II, which reads the nucleotides on the DNA and synthesizes the complementary mRNA strand.

the mRNA are represented as game objects, each with a set of Boolean values that determine which state the mRNA is in and what its subsequent actions will be. For instance, THO will bind to the mRNA first, and only bind to mRNA without any other export factors attached to it (Fig. 8 8 ). Similarly, the UAP56 protein only binds to mRNA if THO is attached (Fig. 8p). Using these states, the order in which the export factors bind to mRNA can be controlled in Unity. We have reproduced this binding process such that THO binds to the mRNA first, followed by UAP56 and ALY (Fig. $8 \mathrm{c}$ and d). When ALY has bound, it recruits the Nxf1-Nxt1 complex (Fig. 8p), which tags the mRNA and pulls it to the nearest nuclear pore (Fig. 99 where it is transported into the cytoplasm and can undergo translation for protein synthesis (Fig. 8f).

By illustrating mRNA export in an immersive 3D space, one can gain a better view of the spatial arrangements and locations of the molecular entities involved in these processes. As humans, we are good at comprehending 3D spaces that we can explore by walking through, exploring, and inspecting objects. Changing the camera view and looking closer at the structures one can identify their functional subunits (binding sites), their relative sizes and other information (e.g. interaction partners) in a rather intuitive manner. Owing to this interactivity, Eukaryo is able to visually convey how the different export factors and signals interact with the mRNA, as well as clearly portray where in a cell these processes occur.

\section{Microtubule Dynamics}

Together with actin filaments and intermediate filaments, microtubules make up a cell's cytoskeleton (Fig. 12). These filaments consist of a long chain of protein subunits bundled together to increase their structural integrity, thus helping the cell maintain its shape and compartmentalization. Microtubules are the largest filaments, reaching lengths of 50 $\mu \mathrm{m}$ and are part of the filament network that spans across a cell, providing structural support to maintain the cell's shape and its compartmentalization (21). 


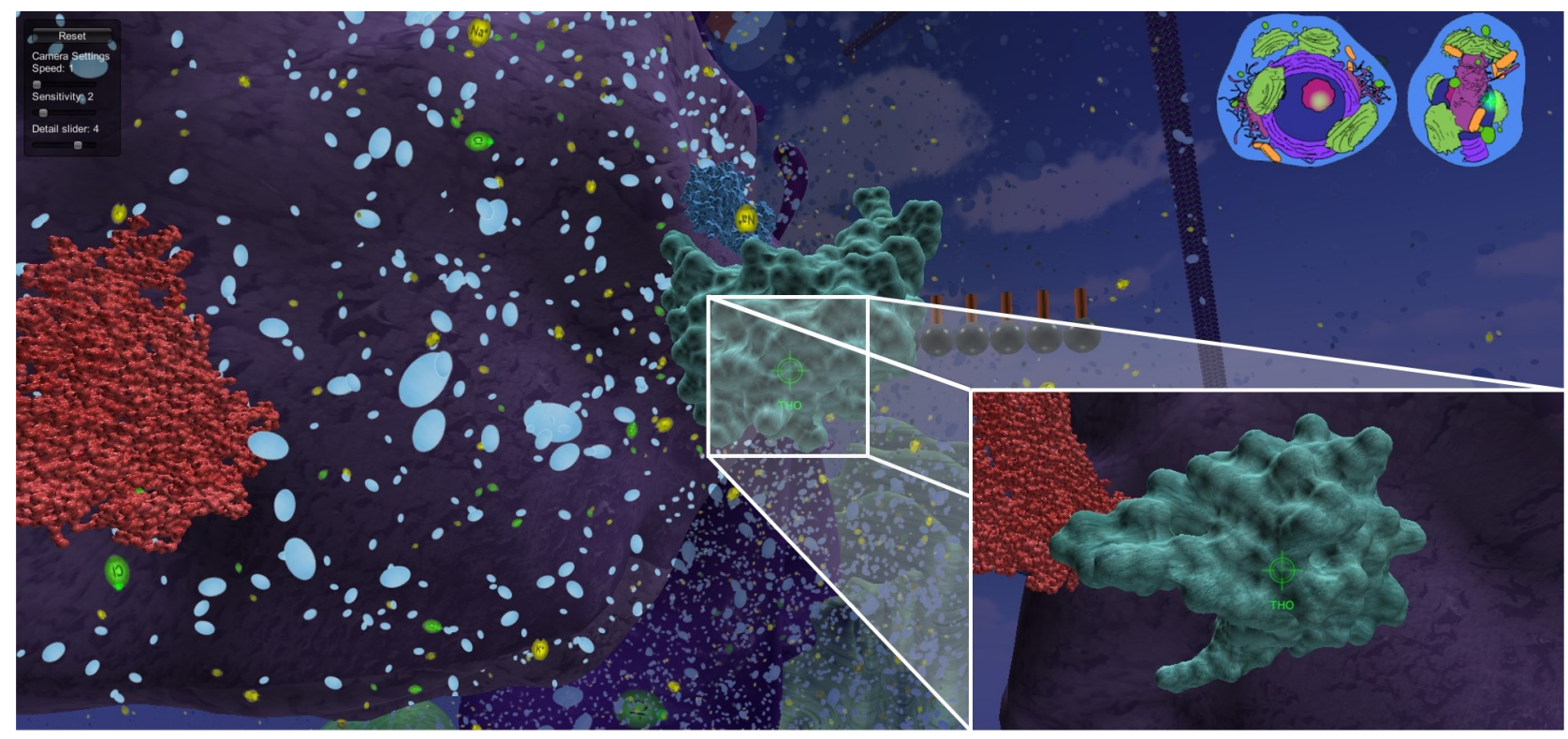

Figure 11: Identifying nuclear export proteins in Eukaryo. By hovering the crosshair over an object, users can readily identify structures and entities within the protein. This scene illustrates all visible entities, including molecules and proteins. These elements can be disabled so attention may be focused on processes of interest. The area of the HUD where this information is displayed is depicted in greater detail within the inset image. All of the controls from Figure 4 are present to facilitate easy adjustment of navigational preferences.

Each microtubule is composed of tubulin, globular proteins that consist of $\alpha$ - and $\beta$-subunits (10). The $\alpha / \beta$ tubulins arrange themselves into a thirteen-membered ring (7, 44). These rings stack on top of one another to form a microtubule (Fig. 15 ). Both $\alpha$ - and $\beta$-tubulin have a binding site for the molecule GTP: in the $\alpha$-subunit, GTP is always present. Following polymerization, the GTP in $\beta$ tubulin is hydrolyzed to GDP. With GTP bound to the $\beta$-subunit in the tubulin dimer, the dimer is known as GTP-tubulin. Similarly, if GTP is hydrolyzed to GDP, then the dimer becomes GDPtubulin. The former favours polymerization, while the latter favours depolymerization; this results in what is known as 'microtubule catastrophe', where the microtubule rapidly disassembles (Fig. 15. Despite their dynamic nature, microtubule growth does not occur in random locations: nucleation and assembly usually begins at microtubule organizing centres (MTOC), organelles that contain $\gamma$-tubulin (7).

These tubulin units are arranged into ring-like structures that act as a template for microtubule growth (Fig. 13). Assembly and disassembly of microtubules are reproduced in Eukaryo: the tubulin units are represented as game objects, and their behaviours are governed by a decision function that selects an action based on the state of the tubulin agent (Fig. 14). Eukaryo models the assembly of tubulin units at the MTOC. In the presence of GTP, tubulin game objects locate and bind to one another using the physics joints to construct the growing microtubule (Fig. 16. Conversely, when GTP is depleted, the tubulin game objects dissociate from one another, falling away from the microtubule until the entire filament has disassembled. Because each of the tubulin game objects are physical entities, they can interact with one another using Unity's physics engine, resulting in emergent behaviours arising within the microtubule structures. For instance, waves propagate through the protofilaments making up the microtubule. In Eukaryo, these result from the collisions between the tubulin game objects, but in a biological cell, this phenomenon is thought to arise from energy released when GTP is hydrolysed to GDP during assembly in the cytosol (Fig. 18) (37).

Moreover, the effects of cytosol viscosity on the rate of assembly is implemented; higher viscosity values correspond with an increased protein density in the cytosol, resulting in faster assembly by increasing the probability that the tubulin proteins can collide with and interact with the growing end of the microtubule (45). Similarly, a reduced viscosity lowers the assembly rates. These values can be controlled in Eukaryo, enabling users to visually observe the effects of different environmental conditions on how microtubules assemble. It was found that altering the cytosol viscosity has an impact on how assembly proceeds: increasing the assembly speed results in the ends becoming forked. Conversely, lower viscosity values result in the microtubules growing more slowly but also increases the likelihood that the protofilaments become tangled with one another (Fig. 18).

The navigational systems in Eukaryo make it easy to observe the assembly/disassembly processes from different perspectives, both very close at a high resolution of detail, and further away to capture the larger structures. By immersing users into a space where they may view and interact with the model, Eukaryo is able to convey the spatial arrangement and the structure building processes just like observing beams and struts in a 3-dimensional architectural model, only that man-made architecture does not self- 


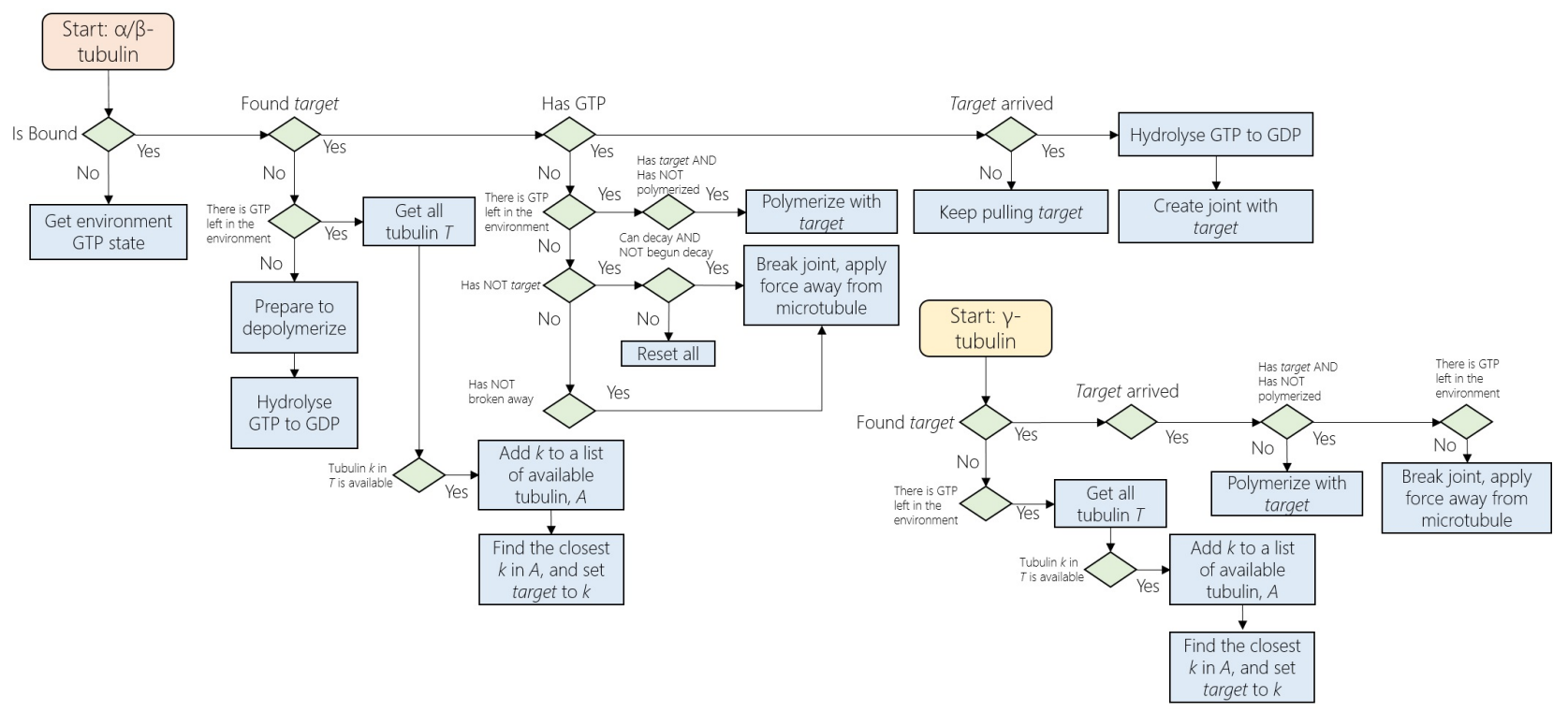

Figure 14: Tubulin decision functions for $\alpha / \beta$-tubulin (orange) and $\gamma$-tubulin (yellow). The decision function is used to determine which actions should be performed given a particular state at each game engine iteration. Here, target refers to the nearest tubulin to pull in (the tubulin agent marked in blue in Fig. 16 .

assemble.

\section{Eukaryo for HoloLens}

In virtual reality (VR) applications, the real world is replaced by virtual, simulated worlds. In augmented reality (AR), however, display technologies supplement, or augment, our real world and its physical objects. Through AR, one can provide additional elements that deliver complementing information about the real world and, in some cases, offer a modicum of interactivity (15).

AR devices come in several forms, with the most common being hand-held devices such as smart phones and wearable headsets (e.g., (31)). Microsoft's HoloLens is another, more recent example of an AR headset (32). Released in January 2016, the HoloLens is able to project high-resolution images and is equipped with a camera for tracking hand motions. In combination with spoken commands, it thus allows for a wide range of gestures to interact with the virtual elements. Scenes constructed in Unity can be exported into HoloLens (42). We have modified Eukaryo so that it can run on HoloLens. Our implementation, HoloCell, is a first prototype of an AR-based cell model, which allows us to explore molecular worlds through innovative ways, including the integration of physical spaces into the actual simulations (Fig. 19).

In HoloCell, the Eukaryo model is presented as a cell that users can place in the middle of a room, walk around, inspect organelles, request more information on each entity, and enter some of the cellular sub-spaces. For example, one can inspect elements of the metabolic processes inside the mitochondria. Interacting with different organelles and proteins in the cell is accomplished using hand gestures and/or voice commands (Fig. 20). Additionally, the cell is responsive to a user's distance to certain elements: the model expands to fill a room, thus placing the user inside the cell; similarly, the cell recedes when one steps away from it. HoloCell and AR represent an exciting direction for implementing additional features for navigation, story telling, and modes of interactivity for Eukaryoand other complex, dynamic scenarios.

\section{Conclusions}

We have implemented Eukaryo as an interactive, 3D virtual cell that models key structures and molecular interactions within a eukaryotic cell. Eukaryo allows users to immerse themselves inside a cell and gain an understanding of the scope and scale of biomolecular interactions. Using Unity and MiddleVR, Eukaryo can be deployed on a range of VR environments. We have tested Eukaryo in a CAVE and with the Oculus Rift head mounted display. For navigation and other controls, we use a commercially-available gamepad controller. We have also implemented a simplified model of Eukaryo on the HoloLens.

Eukaryo's ease-of-use and versatility make it suited for biological education in the classroom, in science centres, or at home using a game station. We believe that interactive 3D models like Eukaryo can be used to supplement existing teaching methods, immersing students within a cell to confer more memorable, illustrative learning experiences about the basic building blocks of life. 


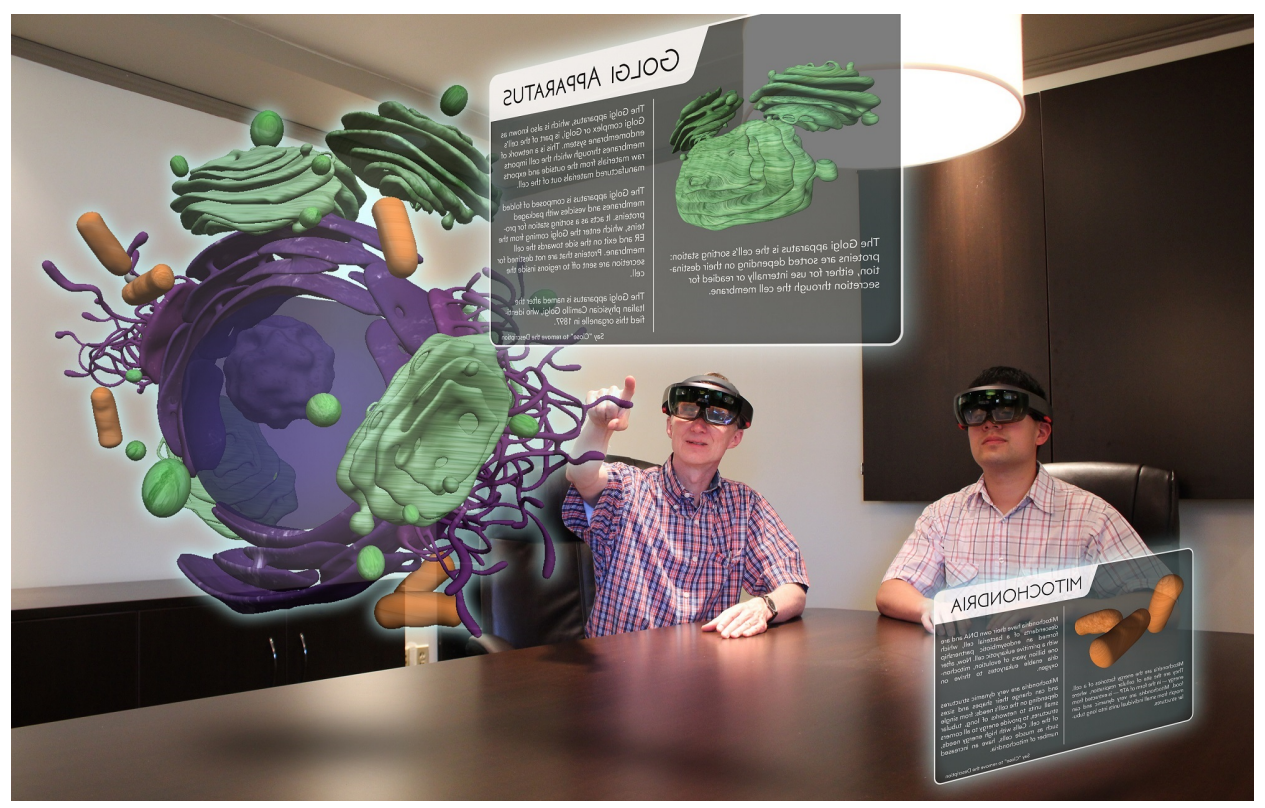

Figure 19: An illustration of how Eukaryo can be deployed in a room with several users. The AR environment allows multiple users to view the same scene, making it easy to accommodate for collaboration.

\section{REFERENCES}

[1] Alberts, B. and Johnson, A. and Lewis, J. and Raff, M. and Roberts, K. and Walter, P. Molecular Biology of the Cell. Garland Science, Taylor \& Francis Group, LLC, sixth edition, 2015.

[2] I. Asimov. Fantastic Voyage (novel). Bantam, 1988.

[3] BioVisions. The inner life of the cell (video).

[4] I. Burleigh et al. DNA in action! A 3d swarm-based model of a gene regulatory system. In First Australian Conference on Artificial Life, Canberra, AU, 2003.

[5] S. R. Carmody and S. R. Wente. mRNA nuclear export at a glance. Journal of cell science, 122(Pt 12):1933-7, June 2009.

[6] C. Cruz-Neira et al. Surround-screen projection-based virtual reality: the design and implementation of the CAVE. 20th annual conference on Computer graphics and interactive techniques, pages 135-142, 1993.

[7] A. Desai and T. J. Mitchison. Microtubule polymerization dynamics. Annual review of cell and developmental biology, 13:83-117, 1997.

[8] A. Esmaeili et al. PROKARYO: an illustrative and interactive computational model of the lactose operon in the bacterium Escherichia coli. BMC Bioinformatics, 16(1):311, 2015.

[9] R. Fleischer. Fantastic Voyage (film). Twentieth Century Fox Film Corporation, 1966.

[10] D. A. Fletcher and R. D. Mullins. Cell mechanics and the cytoskeleton. Nature, 463(7280):485-492, 2010.

[11] E. Games. Unreal engine 4 documentation, 2015.
[12] Gáspár Jékely, editor. Eukaryotic Membranes and Cytoskeleton, volume 607. Springer-Verlag New York, New York, 1 edition, 2007.

[13] D. S. Goodsell. The Machinery of Life. Springer New York, 2nd edition, 2009.

[14] D. S. Goodsell. Miniseries: Illustrating the machinery of life: Eukaryotic cell panorama. Biochemistry and Molecular Biology Education, 39(2):91-101, 2011.

[15] M. Graham, M. Zook, and A. Boulton. Augmented reality in urban places: contested content and the duplicity of code. Transactions of the Institute of British Geographers, 38(3):464-479, 2013.

[16] J. W. Haefner. Modeling Biological Systems:: Principles and Applications. Springer Science \& Business Media, 2005.

[17] C. Jacob and I. Burleigh. Biomolecular swarms: an agent-based model of the lactose operon. Natural Computing, 3(4):361-376, 2004.

[18] C. Jacob and I. Burleigh. Genetic programming inside a cell. Genetic Programming Theory and Practice III, 9:191-206, 2006.

[19] C. Jacob et al. Swarms and genes: Exploring $\lambda$-switch gene regulation through swarm intelligence. In IEEE Congress on Evolutionary Computation, 2006.

[20] C. Jacob et al. LINDSAY Virtual Human. In J. Kolodziej et al., editors, Advances in Intelligent Modelling and Simulation, pages 327-349. Springer Heidelberg, Berlin, 2012.

[21] G. Jékely. Eukaryotic Membranes and Cytoskeleton, volume 607, pages 111-8. Springer, New York, 1st edition, 2007. 


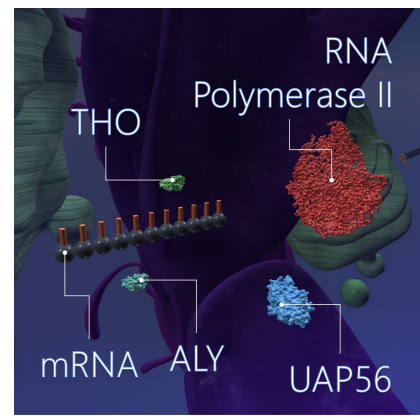

(a) Nascent mRNA

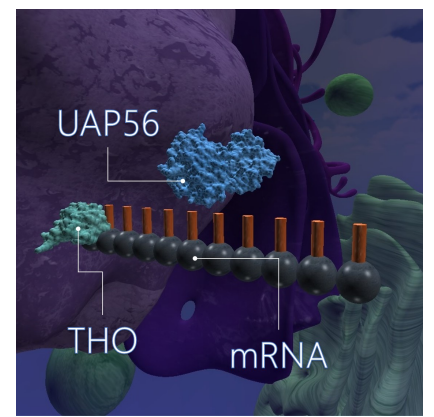

(c) UAP56 binds

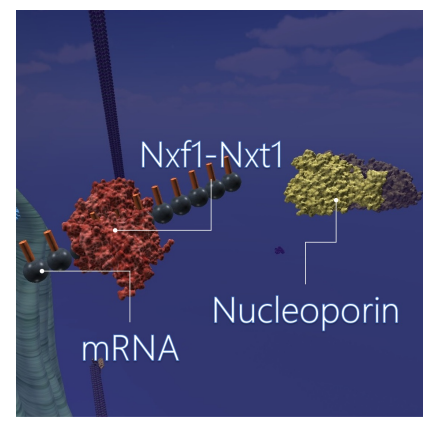

(e) Nxf1-Nxt1 binds

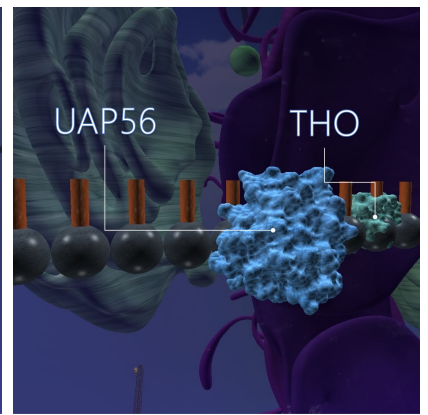

(b) THO binds

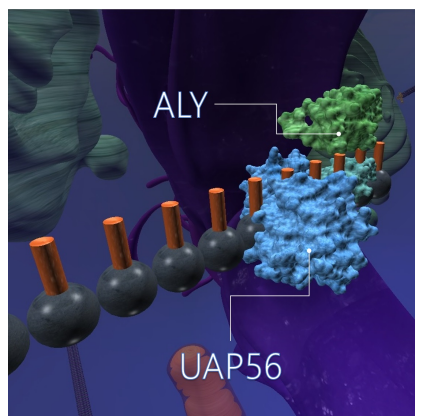

(d) ALY binds

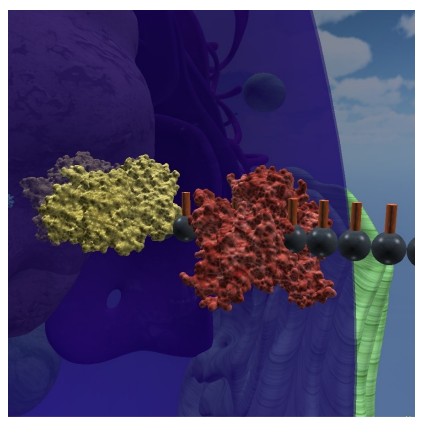

(f) Export across NPC
Figure 8: Binding of mRNA export factors result in mRNA export from nucleus into the cytoplasm.

[22] T. Kuhlen and B. Hentschel. Quo vadis cave: does immersive visualization still matter? IEEE computer graphics and applications, (5):14-21, 2014.

[23] S. Kuntz. MiddleVR: A generic VR toolbox. IEEE Virtual Reality Conference, pages 391-392, 2015.

[24] B. Laha et al. Effects of immersion on visual analysis of volume data. Visualization and Computer Graphics, IEEE Transactions on, 18(4):597-606, 2012.

[25] B. Laha et al. Effects of vr system fidelity on analyzing isosurface visualization of volume datasets. IEEE Transactions on Visualization and Computer Graphics, 20:513-522, 04/2014 2014.

[26] B. Laha et al. Effects of vr system fidelity on analyzing

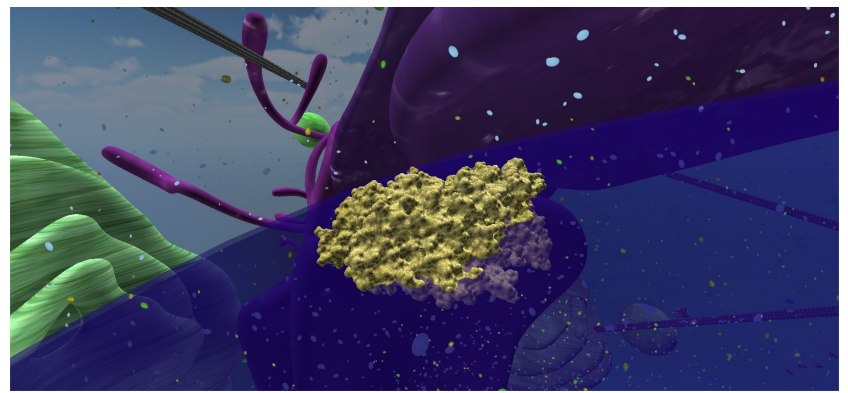

Figure 9: Close-up view of the nucleoporin, also known as the nuclear pore complex (NPC). These pores dot the nuclear surface and act as pathways for the controlled import and export of materials into the nucleus.

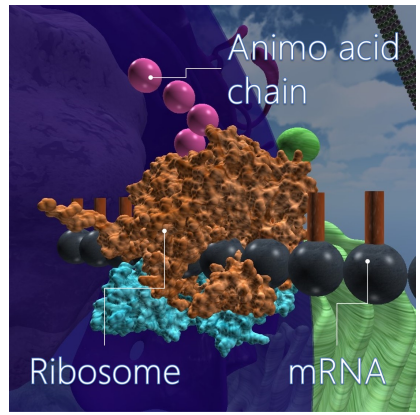

(a) Nascent amino acid strand

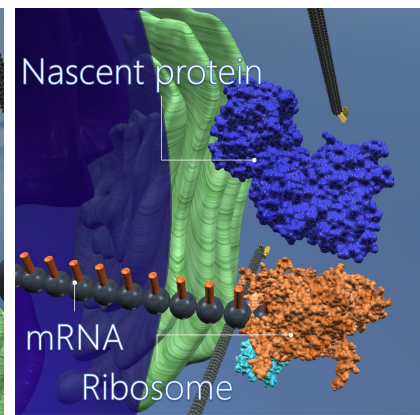

(b) Newly-synthesized protein
Figure 10: Translation of mRNA by ribosome protein. (a) The ribosome creates a strand of amino acids from the code specified on the mRNA, which (b) folds into a new protein.

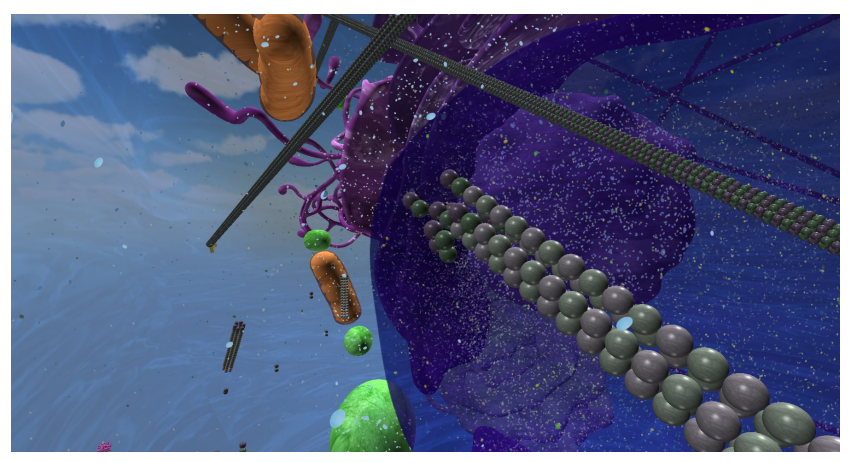

Figure 12: Microtubules are the rod-like structures that form a cell's skeleton and act as transportation highways.

isosurface visualization of volume datasets. Visualization and Computer Graphics, IEEE Transactions on, 20(4):513-522, 2014.

[27] Lodish, H.F. and Berk, A. and Kaiser, C.A. and Krieger, M. and Bretscher, A. and Ploegh, $\mathrm{H}$ and Amon, A. Molecular Cell Biology. Number 1. WH Freeman and Company, New York, New York, USA, seventh edition, 2014. 


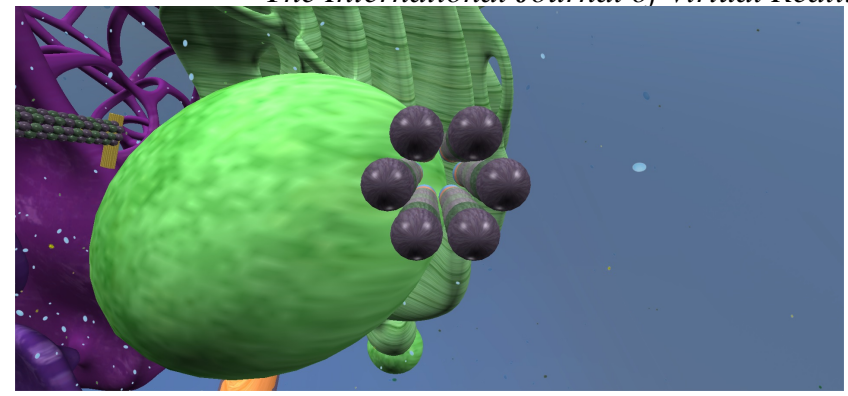

Figure 13: A cross section of the microtubule illustrates their ring-like arrangement. Microtubules are hollow in their center, and while we have chosen to model them as a sixmembered ring in Eukaryo, microtubules in biological systems are thirteen-membered rings.

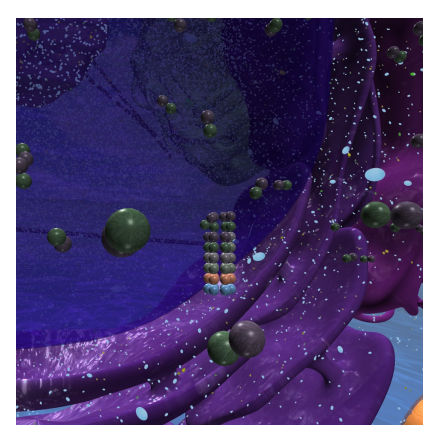

(a) Initialization of assembly

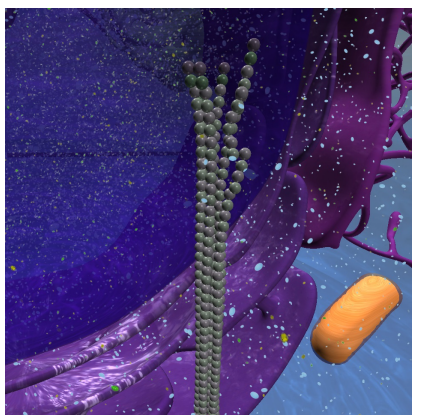

(c) Depolymerization begins

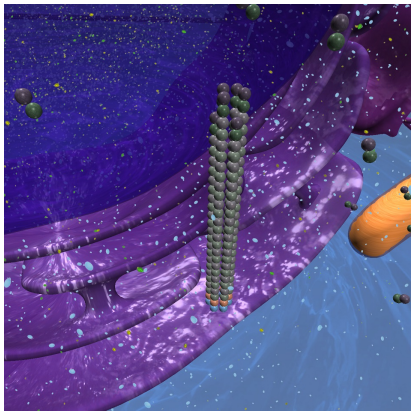

(b) Microtubule elongation

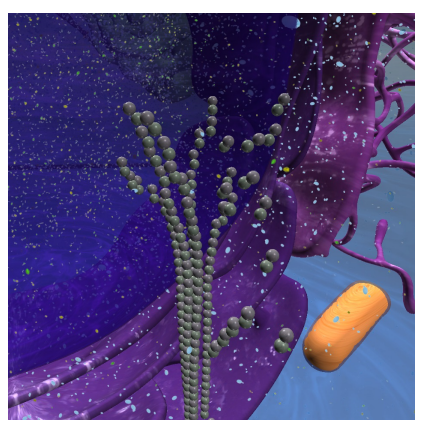

(d) "Catastrophe"
Figure 15: Microtubule assembly and disassembly.

[28] L. M. Loew and J. C. Schaff. The virtual cell: a software environment for computational cell biology. Trends in biotechnology, 19(10):401-6, Oct. 2001.

[29] Z. Lv et al. Game on, science - how video game technology may help biologists tackle visualization challenges. PLoS ONE, 8(3):e57990, 032013.

[30] Maya. autodesk.com.

[31] Meta. Meta spaceglasses, www.getameta.com 2015.

[32] Microsoft. microsoft.com

[33] Middle VR User Guide. middlevr.com 2012.

[34] M. Norrby. Molecularrift, a gesture based interaction tool for controlling molecules in 3-d. 2015.

[35] Oculus Rift. WwW.oculus.com, 2015.

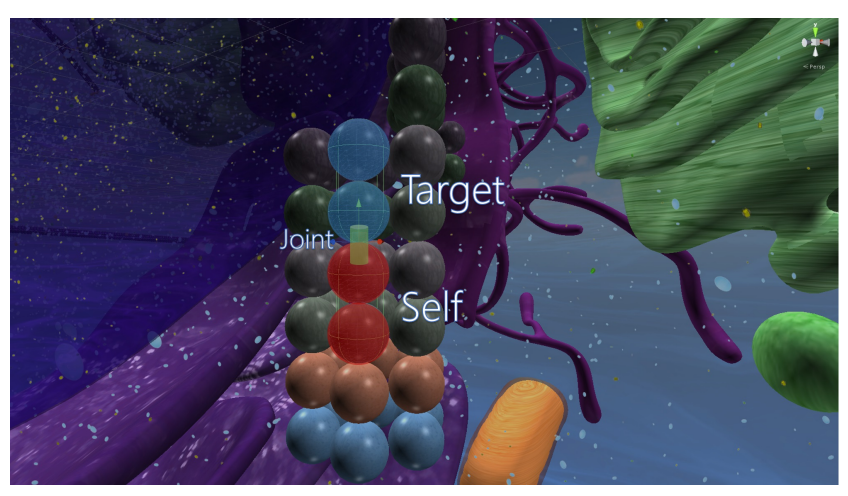

Figure 16: A tubulin agent (red) with its target (blue) and a physics joint (green) connecting the two entities. The target tubulin is a reference to the nearest available tubulin agent. All of the tubulin game objects can physically interact with one another. The physics joint is a Unity component that constrains two objects together. This joint is created after the target has reached its destination and is configured to behave like a spring.

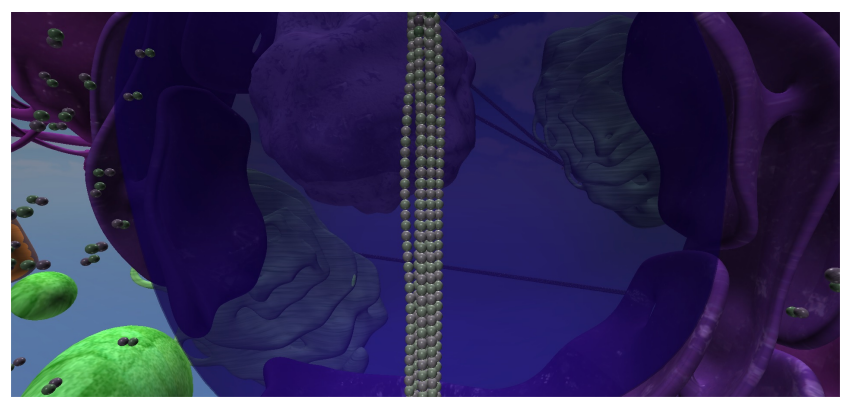

Figure 17: Small waves propagate through the protofilaments making up the microtubule. This effect arises purely from the interactions among the tubulin game objects in $E$ karyo.

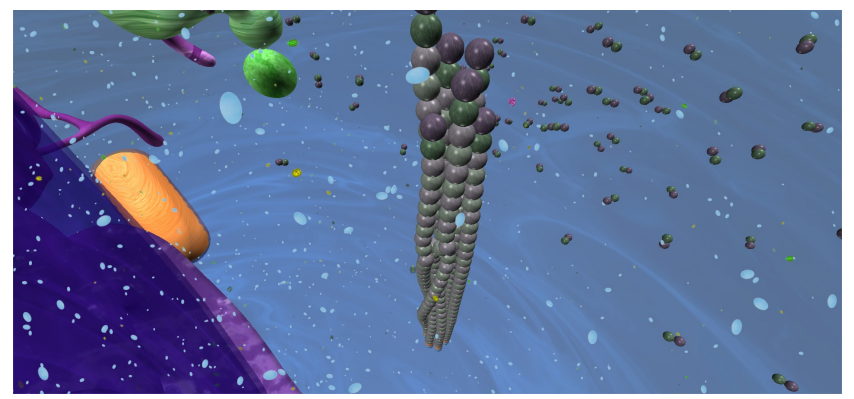

Figure 18: Protofilaments tangling in a simulated microtubule. The tangled filaments arise as a result of the tubulin game objects interacting with one another.

[36] E. F. Pettersen, T. D. Goddard, C. C. Huang, G. S. Couch, D. M. Greenblatt, E. C. Meng, and T. E. Ferrin. UCSF Chimera - A visualization system for exploratory research and analysis. Journal of Computational Chemistry, 25(13):1605-1612, 2004. 


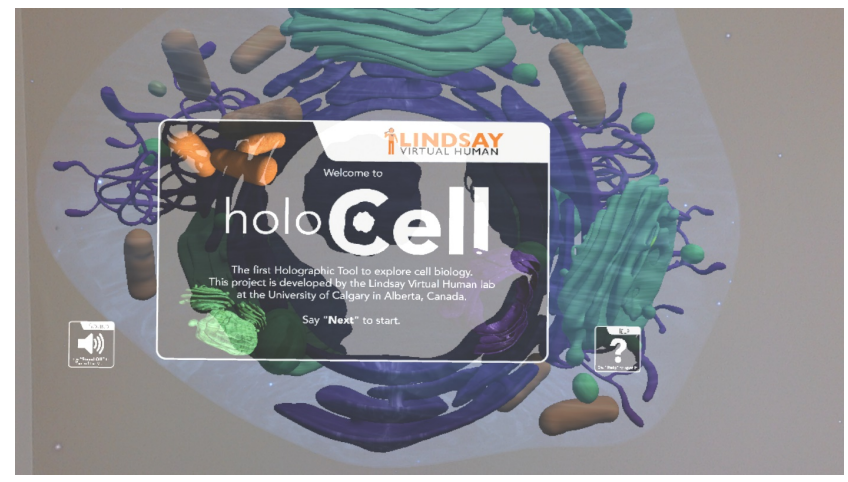

Figure 20: Eukaryo running on the HoloLens. This version, which we call HoloCell, makes use of gestures and audio commands for navigation.

[37] J. Pokorný. Excitation of vibrations in microtubules in living cells. Bioelectrochemistry, 63(1-2):321-326, 2004.

[38] E. Ragan et al. Studying the effects of stereo, head tracking, and field of regard on a small-scale spatial judgment task. Visualization and Computer Graphics, IEEE Transactions on, 19(5):886-896, 2013.

[39] W. T. Reeves. Particle systems-a technique for modeling a class of fuzzy objects. ACM SIGGRAPH Computer Graphics, 17(3):359-375, 1983.

[40] V. Sarpe and C. Jacob. Simulating the decentralized processes of the human immune system in a virtual anatomy model. BMC Bioinformatics, 14(Suppl 6):126, 2013.

[41] M. Tomita et al. E-cell: software environment for whole-cell simulation. Bioinformatics, 15(1):72-84, Jan. 1999.

[42] Unity Technologies. Unity Manual, 2012.

[43] J. D. Westbrook and P. M. D. Fitzgerald. The pdb format, mmcif formats, and other data formats. In P. E. Bourne and H. Weissig, editors, Structural Bioinformatics, chapter 8. Wiley-Liss, 2 edition, 2003.

[44] B. Wickstead and K. Gull. The evolution of the cytoskeleton. Journal of Cell Biology, 194(4):513-525, 2011.

[45] M. Wieczorek, S. Chaaban, and G. J. Brouhard. Macromolecular crowding pushes catalyzed microtubule growth to near the theoretical limit. Cellular and Molecular Bioengineering, 6(4):383-392, 2013.

[46] Zygote Media Group. zygote.com, 2015. 